\title{
Casimir-Polder potential and transition rate in resonating cylindrical cavities
}

\author{
Simen Å. Ellingsen \\ Department of Energy and Process Engineering, Norwegian University of Science and Technology, N-7491 Trondheim, Norway \\ Stefan Yoshi Buhmann and Stefan Scheel \\ Quantum Optics and Laser Science, Blackett Laboratory, Imperial College London, \\ Prince Consort Road, London SW7 2AZ, United Kingdom
}

(Dated: October 31, 2019)

\begin{abstract}
We consider the Casimir-Polder potential of particles placed inside a metallic cylindrical cavity at finite temperatures, taking account of thermal non-equilibrium effects. In particular, we study how the resonant (thermal non-equilibrium) potential and transition rates can be enhanced by finetuning the radius of the cavity to match the transition wavelength of the dominant transition of the particle. Numerical calculations show that the CP potential acting atoms prepared in low-lying Rydberg states can be enhanced beyond $30 \mathrm{kHz}$, which is within the range of observability of modern experiments. Because the magnitude of the resonance peaks depend sensitively on the low frequency dissipation of the cavity metal, experiments in this set-up could be a critical test of the disputed thermal correction to the Casimir force between metal plates.
\end{abstract}

PACS numbers: $34.35 .+\mathrm{a}, 12.20 .-\mathrm{m}, 42.50 . \mathrm{Ct}, 42.50 . \mathrm{Nn}$

\section{INTRODUCTION}

Casimir-Polder (CP) forces [1] belong to the group of of dispersion forces which arise due to the fluctuations of the quantized electromagnetic field. They occur between polarizable atoms or molecules and metallic or dielectric macroscopic bodies and can be intuitively thought of as the dipole-dipole force caused by spontaneous and mutually correlated polarization of the atom or molecule and the matter comprising the body. Under the assumption of thermal equilibrium, $\mathrm{CP}$ forces have been commonly investigated in the linear-response formalism [2-4].

Recent theoretical predictions [5] as well as experimental realizations [6] for $\mathrm{CP}$ forces in thermal nonequilibrium situations have pointed towards interesting effects which arise when an atom at equilibrium with its local environment interacts with a body held at a different temperature. In particular, depending on the temperatures of the macroscopic body and the environment, the force can change its character from being attractive to repulsive and vice versa.

Non-equilibrium situations between atom and local environment can be investigated by means of normal-mode quantum electrodynamics (QED) [7, 8] or macroscopic QED in absorbing and dispersing media $[9,10]$. In this case, thermal excitation and de-excitation processes lead to resonant contributions to the force [11] (cf. similar findings reported in Ref. [12]). At particle-body separations that are larger than the wavelengths associated with the dominating atomic transitions (retarded regime), the interaction potential becomes spatially oscillating [13]. Similar behaviour has been observed for the transition rate of molecules in the past $[14,15]$.

The spatially oscillating non-equilibrium forces on ground-state atoms or molecules are proportional to the thermal photon number. In order to observe them, it is necessary to make use of atomic systems whose inter-

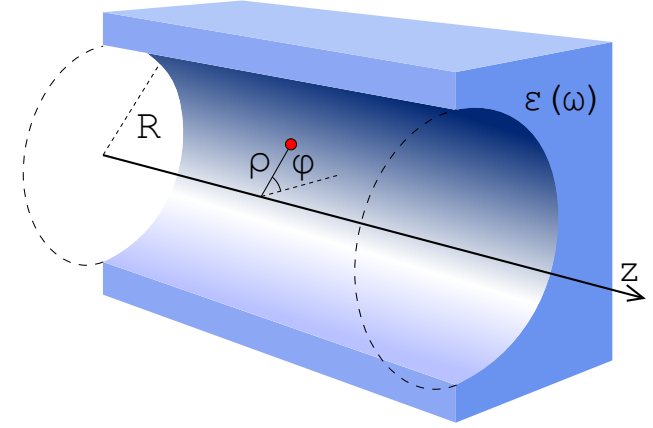

FIG. 1: Cross-section of the geometry considered: a particle in a vacuum-filled circularly cylindrical cavity.

nal eigenstates exhibit energy separations of order $k_{\mathrm{B}} T$ or less ( $T$ : temperature, $k_{\mathrm{B}}$ : Boltzmann constant). We have investigated polar molecules with their low-energy rotational and vibrational transitions as possible candidates [13]. However, the large photon numbers obtained using molecules with small excitation energies come at the cost: Due to the large wavelengths associated with such molecular transitions, the retarded regime where oscillations might be observed sets in at very large distances, typically of the the order of tens to hundreds of microns. As the CP potential decays away rapidly from the body surface, it is very small at such distances.

As already discussed in conjunction with excited atoms in a zero temperature environment, resonant forces can be enhanced in a planar cavity whose width is finetuned to match the wavelength of the transition [16-22]. Note that resonating cavities have been employed experimentally for enhancement and inhibition of spontaneous emission rates for excited systems for a long time (cf. e.g. [29-32]). We have previously investigated the potential of such a setup to enhance the predicted spatial oscillations of the thermal force on ground-state molecules [23]. 
Unfortunately, the cavity enhancement factor turns out to scale logarithmically with the cavity $Q$ factor, which strongly limits the possibilities of the scheme. As we have shown, it is unlikely to achieve more than an order of magnitude's enhancement of the force amplitudes, which is still insufficient for detection using polar molecules.

In the present article, we present a scheme by which oscillations of the resonant thermal $\mathrm{CP}$ force can be brought into the measurable regime. This is achieved by replacing the planar cavity with a cylindrical cavity [24] and employing Rydberg atoms rather than polar molecules [25]. The geometry is shown in Fig. 1. The limited cavity enhancement in a planar setup is due to the insufficient, purely one-dimensional confinement of the electromagnetic modes. A cylindrical cavity is therefore an obvious candidate for improvement: It confines the modes in two out of three spatial dimensions, so that a stronger resonant enhancement may be expected. At the same time, it is a practical geometry for experimental and guiding purposes, allowing particles to travel freely along the axial direction (we will only consider particles at rest herein, leaving moving atoms [26] in this geometry for future study). As a caveat, one has to bear in mind that a cylindrical cavity will simultaneously enhance both the $\mathrm{CP}$ potential and the relaxation rates; thus reducing the time scales on which non-equilibrium effects can be observed. A similar geometry, i.e., an (anisotropic) particle above a spherical hole in a thin metal plate, has recently been suggested as a candidate for observing repulsive Casimir forces [27].

Rydberg atoms are ideal candidates for observing resonant thermal CP potentials. Their valence electrons are excited to relatively stable states with very large principal quantum numbers $n$, typically in the range 30-60. The spatial extent of such highly excited atoms is then enormous on an atomic scale, exceeding a micron in diameter, and the transition dipole moments consequently orders of magnitude larger than those of ground-state atoms or polar molecules. Resonant CP interactions being proportional to the respective transition dipole moment squared, a strong enhancement follows. Moreover, an atom excited to a particular eigenstate with large $n$ is necessarily out of thermal equilibrium with its environment, and the energy difference to neighbouring states is typically small compared to $k_{\mathrm{B}} T$ at room temperature, hence fulfilling the condition for observing oscillating potential. Remarkably, we recently found that the same property ensures that the CP potential in a Rydberg atom is virtually independent of temperature from room temperature down to absolute zero [28].

Outline of the paper. The structure of the paper is as follows. In section II we present the general formalism of the thermal CP interaction and transition rates in a cylindrical cavity, including the necessary Green tensor. Thereafter, in section III we derive the cylinder radii which resonate with the atomic transition frequency. We begin with the simplest case of perfectly conducting cavity and then discuss how the optimal radii deviate from the perfect conductor results when realistic metallic permittivity functions are employed. We provide simple formulas for the optimal radii when the permittivity of the cylinder medium is large. In section IV, we discuss how the potential and heating rate enhancements scale with the relevant atomic and cavity parameters, with emphasis on the dependence on the cavity permittivity. Finally we undertake numerical calculations of resonant $\mathrm{CP}$ potential and heating rates for two example cases: the $32 \mathrm{~s}_{1 / 2} \rightarrow 31 \mathrm{p}_{3 / 2}$ transition of Rydberg Rb in a cylindrical Au cavity at temperature $T=300 \mathrm{~K}$, and a ground state $\mathrm{LiH}$ molecule in a similar cavity.

\section{GENERAL FORMALISM}

We consider an atomic system (below we study the cases of Rydberg atoms and polar molecules) with internal energy eigenstates $|n\rangle$, eigenenergies $\hbar \omega_{n}$, transition frequencies $\omega_{m n}=\omega_{m}-\omega_{n}$ and dipole matrix elements $\mathbf{d}_{m n}=\langle m|\hat{\mathbf{d}}| n\rangle$, which is prepared in an incoherent superposition of its energy eigenstates with probabilities $p_{n}$.

\section{A. Thermal Casimir-Polder potential}

The thermal CP force on such a system in an environment of uniform temperature $T$ was derived in detail in Ref. [11]. As shown, it is conservative in the perturbative limit, $\mathbf{F}(\mathbf{r})=-\nabla U(\mathbf{r})$, where the associated $\mathrm{CP}$ potential is given by

$$
U(\mathbf{r})=\sum_{n} p_{n} U_{n}(\mathbf{r})
$$

The potential components associated with a given eigenstate $n$ splits naturally into a non-resonant contribution $U_{n}^{\mathrm{nr}}$ and a resonant one $U_{n}^{\text {res }}$,

$$
U_{n}(\mathbf{r})=U_{n}^{\mathrm{nr}}(\mathbf{r})+U_{n}^{\mathrm{res}}(\mathbf{r}) .
$$

The non-resonant potential $U_{n}^{\mathrm{nr}}$ is due to virtual photons and is reminiscent of that obtained by a dilute-gas expansion of Lifshitz' formula [2]. The resonant contribution $U_{n}^{\text {res }}$ is due to absorption and emission of thermal photons; it is present because the particle in its ground state is out of thermal equilibrium with its environment.

The non-resonant potential reads

$$
U_{n}^{\mathrm{nr}}(\mathbf{r})=\frac{k_{\mathrm{B}} T}{\varepsilon_{0}} \sum_{j=0}^{\infty} \frac{\xi_{j}^{2}}{c^{2}} \operatorname{Tr}\left[\boldsymbol{\alpha}_{n}\left(i \xi_{j}\right) \cdot \mathbf{G}^{(1)}\left(\mathbf{r}, \mathbf{r}, i \xi_{j}\right)\right]
$$

where $\mu_{0}$ is the free-space permeability, $\xi_{j}=2 \pi j k_{\mathrm{B}} T / \hbar$ is the $j$ th Matsubara frequency, and the prime on the Matsubara sum indicates that the $j=0$ term is to be taken with half weight. The atomic/molecular polarizability is 
given by

$$
\boldsymbol{\alpha}_{n}(\omega)=\lim _{\epsilon \rightarrow 0} \frac{1}{\hbar} \sum_{k}\left[\frac{\mathbf{d}_{k n} \mathbf{d}_{n k}}{\omega+\omega_{k n}+i \epsilon}-\frac{\mathbf{d}_{n k} \mathbf{d}_{k n}}{\omega-\omega_{k n}+i \epsilon}\right]
$$

and $\mathbf{G}^{(1)}\left(\mathbf{r}, \mathbf{r}^{\prime}, \omega\right)$ is the scattering part of the classical Green tensor of the geometry the particle is placed in. Note that dyadic multiplication is implied for products of vectors without multiplication symbol: $\mathbf{A B} \equiv \mathbf{A} \otimes$ B. For an isotropic particle, the non-resonant potential components may be simplified to

$$
U_{n}^{\mathrm{nr}}(\mathbf{r})=\frac{k_{\mathrm{B}} T}{\varepsilon_{0}} \sum_{j=0}^{\infty} \frac{\xi_{j}^{2}}{c^{2}} \alpha_{n}\left(i \xi_{j}\right) \operatorname{Tr} \mathbf{G}^{(1)}\left(\mathbf{r}, \mathbf{r}, i \xi_{j}\right)
$$

with

$$
\alpha_{n}(\omega)=\lim _{\epsilon \rightarrow 0} \frac{2}{3 \hbar} \sum_{k} \frac{\left|\mathbf{d}_{n k}\right|^{2} \omega_{k n}}{\omega_{k n}^{2}-\omega^{2}-i \epsilon \omega}
$$

The resonant potential reads

$$
\begin{gathered}
U_{n}^{\mathrm{res}}(\mathbf{r})=\mu_{0} \sum_{k} \omega_{k n}^{2} \mathbf{d}_{n k} \cdot \operatorname{Re}^{(1)}\left(\mathbf{r}, \mathbf{r},\left|\omega_{k n}\right|\right) \cdot \mathbf{d}_{k n} \\
\times\left\{\Theta\left(\omega_{k n}\right) n\left(\omega_{k n}\right)-\Theta\left(\omega_{n k}\right)\left[n\left(\omega_{n k}\right)+1\right]\right\}
\end{gathered}
$$

where $\mu_{0}$ is the free-space permeability, and $\Theta(x)$ denotes the Heaviside step function. The photon number follows the Bose-Einstein distribution

$$
n(\omega)=\left[\exp \left(\frac{\hbar \omega}{k_{\mathrm{B}} T}\right)-1\right]^{-1}
$$

For an isotropic particle, the resonant potential components reduce to

$$
\begin{aligned}
& U_{n}^{\mathrm{res}}(\mathbf{r})=\frac{\mu_{0}}{3} \sum_{k} \omega_{k n}^{2}\left|\mathbf{d}_{n k}\right|^{2} \operatorname{TrRe} \mathbf{G}^{(1)}\left(\mathbf{r}, \mathbf{r},\left|\omega_{k n}\right|\right) \\
& \times\left\{\Theta\left(\omega_{k n}\right) n\left(\omega_{k n}\right)-\Theta\left(\omega_{n k}\right)\left[n\left(\omega_{n k}\right)+1\right]\right\} .
\end{aligned}
$$

\section{B. Environment-assisted transition rates}

An atomic system intially prepared in a given energy eigenstate $|n\rangle$ and placed in an environment of uniform temperature $T$ will undergo transitions to different eigenstates due to an absorption and emission of thermal photons. As shown in Ref. [41], the total rate

$$
\Gamma_{n}(\mathbf{r})=\Gamma_{n}^{(0)}+\Gamma_{n}^{(1)}(\mathbf{r})
$$

for transitions out of state $|n\rangle$ consists of a free-space part $\Gamma_{n}^{(0)}$ and an environment-induced part $\Gamma_{n}^{(1)}$. In the perturbative limit, these are given as

$$
\begin{aligned}
\Gamma_{n}^{(0)}= & \sum_{k} \frac{\left|\omega_{k n}\right|^{3}\left|\mathbf{d}_{n k}\right|^{2}}{3 \pi \hbar \varepsilon_{0} \hbar}\left\{\Theta\left(\omega_{n k}\right)\left[n\left(\omega_{n k}\right)+1\right]\right. \\
& \left.+\Theta\left(\omega_{k n}\right) n\left(\omega_{k n}\right)\right\}
\end{aligned}
$$

and

$$
\begin{aligned}
& \Gamma_{n}^{(1)}(\mathbf{r})=\frac{2}{\varepsilon_{0} \hbar} \sum_{k} \frac{\omega_{k n}^{2}}{c^{2}} \mathbf{d}_{n k} \cdot \operatorname{Im} \mathbf{G}^{(1)}\left(\mathbf{r}, \mathbf{r},\left|\omega_{k n}\right|\right) \cdot \mathbf{d}_{k n} \\
& \times\left\{\Theta\left(\omega_{n k}\right)\left[n\left(\omega_{n k}\right)+1\right]+\Theta\left(\omega_{k n}\right) n\left(\omega_{k n}\right)\right\} \cdot
\end{aligned}
$$

The environment-induced rate simplifies for an isotropic particle to

$$
\begin{gathered}
\Gamma_{n}^{(1)}(\mathbf{r})=\frac{2}{3 \varepsilon_{0} \hbar} \sum_{k} \frac{\omega_{k n}^{2}}{c^{2}}\left|\mathbf{d}_{n k}\right|^{2} \operatorname{Tr} \operatorname{Im} \mathbf{G}^{(1)}\left(\mathbf{r}, \mathbf{r},\left|\omega_{k n}\right|\right) \\
\times\left\{\Theta\left(\omega_{n k}\right)\left[n\left(\omega_{n k}\right)+1\right]+\Theta\left(\omega_{k n}\right) n\left(\omega_{k n}\right)\right\} .
\end{gathered}
$$

\section{The Green tensor in a cylindrical cavity}

We are going to study atomic systems placed at position $\mathbf{r}=(\rho, \varphi, z)$ inside a circularly cylindrical free-space cavity of radius $R$ in a bulk non-magnetic medium with permittivity $\varepsilon=\varepsilon(\omega)$ as shown in Fig. 1. The respective scattering Green can be found in Ref. [33] (see also Appendix A4.2 of [10]):

$$
\begin{aligned}
\mathbf{G}^{(1)}(\mathbf{r}, \mathbf{r}, \omega)= & \frac{i}{4 \pi} \int_{-\infty}^{\infty} d q \sum_{m=0}^{\infty}{ }^{\prime} \eta^{-2}\left[r_{\mathrm{MM}} \mathbf{M}_{o} m \eta(q) \mathbf{M}_{e} m \eta(-q) \pm r_{\mathrm{NM}} \mathbf{N}_{e} m \eta\right. \\
& +r_{\mathrm{NN}} \mathbf{N}_{o}(q) \mathbf{M}_{o} m \eta \\
& (q) \mathbf{N}_{e} m \eta \\
e_{e} m & (-q)]
\end{aligned}
$$


where $\eta=\sqrt{k^{2}-q^{2}}$ and $k=\omega / c$. The cylindrical vector wave functions inside the cavity are $[10,33,34]$ :

$$
\begin{aligned}
& \mathbf{M}_{o} m \eta(q)=\left[\mp \frac{m}{\rho} J_{m}(\eta \rho){ }_{\cos }^{\sin } m \varphi \hat{\varrho}-\eta J_{m}^{\prime}(\eta \rho){ }_{\sin }^{\cos } n \varphi \hat{\boldsymbol{\varphi}}\right] e^{i q z} \\
& \mathbf{N}_{o} m \eta(q)=\left[\frac{i q \eta}{k} J_{m}^{\prime}(\eta \rho)_{\sin }^{\cos } m \varphi \hat{\varrho} \mp \frac{i q m}{k \rho} J_{m}(\eta \rho){ }_{\cos }^{\sin } m \varphi \hat{\boldsymbol{\varphi}}+\frac{\eta^{2}}{k} J(\eta \rho){ }_{\sin }^{\cos } m \varphi \hat{\mathbf{z}}\right] e^{i q z} .
\end{aligned}
$$

The compact wave vector notation used here implies $\mathbf{A}_{o} \mathbf{B}_{e}=\mathbf{A}_{e} \mathbf{B}_{e}+\mathbf{A}_{o} \mathbf{B}_{o}$ etc., and the upper (lower) sign in (2.13) corresponds to upper (lower) index $e, o$ of the vector wave functions. The reflection coefficients $r$ can be found from a system of linear equations as described in Ref. [33]. For the single-interface cylindrical cavity in a bulk medium as considered here, the result for the diagonal coefficients $r_{M} \equiv r_{\mathrm{MM}}$ and $r_{N} \equiv r_{\mathrm{NN}}$ may be written as

$$
r_{M, N}=-\frac{H_{m}^{(1)}(x)}{J_{m}(x)} \tilde{r}_{M, N}
$$

with

$$
\tilde{r}_{\sigma}=\frac{A+B_{\sigma}}{A+B_{D}}, \quad \sigma=M, N
$$

and

$$
\begin{aligned}
A & =-m^{2}(k R)^{2}(q R)^{2}(\varepsilon-1)^{2}, \\
B_{M} & =x_{1}^{2} x^{2}\left[\varepsilon \tilde{h}_{1}^{2} x^{2}-\left(\tilde{h}_{1} \tilde{\jmath}+\varepsilon \tilde{h}_{1} \tilde{h}\right) x_{1} x+\tilde{h} \tilde{\jmath} x_{1}^{2}\right], \\
B_{N} & =x_{1}^{2} x^{2}\left[\varepsilon \tilde{h}_{1}^{2} x^{2}-\left(\varepsilon \tilde{h}_{1} \tilde{\jmath}+\tilde{h}_{1} \tilde{h}\right) x_{1} x+\tilde{h} \tilde{\jmath} x_{1}^{2}\right], \\
B_{D} & =x_{1}^{2} x^{2}\left[\varepsilon \tilde{h}_{1}^{2} x^{2}-(\varepsilon+1) \tilde{h}_{1} \tilde{\jmath} x_{1} x+\tilde{\jmath}^{2} x_{1}^{2}\right] .
\end{aligned}
$$

Here, $x=\eta R, x_{1}=\eta_{1} R, \eta_{1}=\sqrt{\varepsilon(\omega) \omega^{2} / c^{2}-q^{2}}$, $\varepsilon=\varepsilon(\omega)$ is the permittivity of the cylinder and we have defined the shorthand quantities

$$
\tilde{h}=\tilde{h}(x), \quad \tilde{h}_{1}=\tilde{h}\left(x_{1}\right), \quad \tilde{\jmath}=\tilde{\jmath}(x), \quad \tilde{\jmath}_{1}=\tilde{\jmath}\left(x_{1}\right)
$$

where the reduced Bessel functions denoted $\tilde{h}(x)$ and $\tilde{\jmath}(x)$ are

$$
\begin{gathered}
\tilde{h}(x)=\frac{H_{m}^{(1) \prime}(x)}{H_{m}^{(1)}(x)}=\frac{d}{d x} \ln H_{m}^{(1)}(x) ; \\
\tilde{\jmath}(x)=\frac{J_{m}^{\prime}(x)}{J_{m}(x)}=\frac{d}{d x} \ln J_{m}(x) .
\end{gathered}
$$

Note that $\tilde{\jmath}$ is a real function for real arguments whereas $\tilde{h}$ is complex for real arguments. Explicit knowledge of the off-diagonal reflection coefficients is not required; for our purposes, it is sufficient to note that $r_{\mathrm{MN}}=r_{\mathrm{NM}}$ (cf. Refs. [10, 33] for details).

Substituting the vector wave functions (2.14a) and (2.14b) into Eq. (2.13), making use of $r_{\mathrm{MN}}=r_{\mathrm{NM}}$ and the fact that odd functions of $q$ do not contribute to the integral, the Green tensor is found to take the diagonal form

$$
\begin{aligned}
\mathbf{G}^{(1)}(\mathbf{r}, \mathbf{r}, \omega)= & \frac{i}{2 \pi} \int_{0}^{\infty} d q \sum_{m=0}^{\infty}\left\{\left[\frac{m^{2}}{\eta^{2} \rho^{2}} J_{m}^{2}(\eta \rho) r_{M}+\frac{q^{2}}{k^{2}} J_{m}^{\prime 2}(\eta \rho) r_{N}\right] \hat{\varrho} \hat{\varrho}\right. \\
& \left.+\left[J_{m}^{\prime 2}(\eta \rho) r_{M}+\frac{m^{2} q^{2}}{k^{2} \eta^{2} \rho^{2}} J_{m}^{2}(\eta \rho) r_{N}\right] \hat{\boldsymbol{\varphi}} \hat{\boldsymbol{\varphi}}+\frac{\eta^{2}}{k^{2}} J_{m}^{2}(\eta \rho) r_{N} \hat{\mathbf{z}} \hat{\mathbf{z}}\right\} .
\end{aligned}
$$

The trace of the Green tensor required for isotropic molecules hence reads

$$
\begin{aligned}
& \operatorname{Tr} \mathbf{G}^{(1)}(\mathbf{r}, \mathbf{r}, \omega)=\frac{i}{2 \pi} \int_{0}^{\infty} d q \sum_{m=0}^{\infty}\left\{\left(r_{M}+\frac{q^{2}}{k^{2}} r_{N}\right)\right. \\
& \left.\times\left[\frac{m^{2}}{\eta^{2} \rho^{2}} J_{m}^{2}(\eta \rho)+J_{m}^{\prime 2}(\eta \rho)\right]+r_{N} \frac{\eta^{2}}{k^{2}} J_{m}^{2}(\eta \rho)\right\} .
\end{aligned}
$$

It should be noted that the terms containing $m^{2} / \eta^{2} \rho^{2}$ exhibit third-order poles at $q=k$ where $\eta$ vanishes. The physically correct treatment of this pole consists of adding a small imaginary part to the free-space wave vector $k=\omega / c+i \delta$ and performing the limit $\delta \rightarrow 0$. In this way, the pole is circumvented from below as $q$ is integrated along the positive real axis, see Fig. 2(a). Correspondingly, $\eta$ is integrated along a part of the real axis, around the singularity at the origin and along the positive imaginary axis, Fig. 2(c). Recall that in planar geometries the Green tensor at real frequencies may be separated into a propagating part $(q<k)$ which exhibits an oscillating behaviour due to interference of incoming and reflected photons and an evanescent part $(q>k)$ which falls off monotonously away from a surface [13]. In the present case, however, such a distinction cannot 


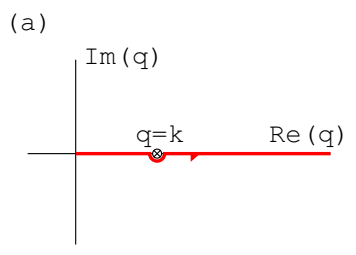

(b)

(c)

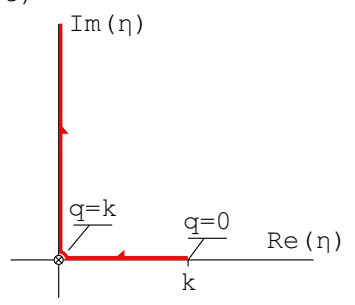

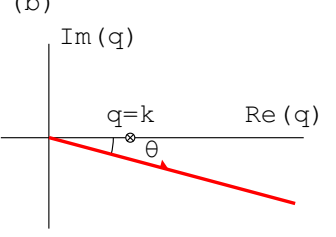

(d)

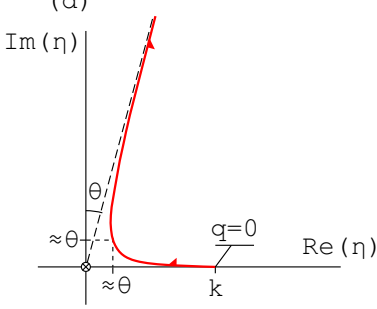

FIG. 2: (a) and (b): Rotation of the $q$-integration path by an angle $\theta$. (c) and (d): Resulting paths for $\eta$.

be made due to the pole at the separation point $q=k$; the individual propagating and evanescent parts would diverge.

For numerical purposes, the original integration contour is unfavourable since it involves subtraction of almost equal numbers, associated with a considerable loss of accuracy. Instead, we rotate the contour of the integral over the variable $q$ to lie along a line at a small negative angle $\theta$, below the real axis as shown in Fig. 2(b). As shown in Fig. 2(d), the corresponding path for $\eta$ is slightly shifted with respect to the orginal one but still contained within the first quadrant: When $\theta \ll 1$, the point of closest approach to the pole at $\eta=0$ is approximately $\theta(1+i)$ and the integration path thence approaches an asymptote at an angle $\pi / 2-\theta$.

We will show in Sec. III B that all the poles of the reflection coefficients lie below the real $x$ axis when $|\varepsilon|<$ $\infty$, approaching the real axis from below in the perfect conductor limit. There are no other possible singularities in the integrands in Eqs. (2.20) and (2.21), so the area contained between the original and deformed integration contours for $\eta$ is free of poles. As the intergands vanish along a path at imaginary infinity connecting the two paths, they are equivalent by virtue of Cauchy's theorem as long as $\theta<\pi / 2$; we choose $\theta=0.1 \mathrm{rad}$. An additional benifit of the rotated integration path is the fact that the integral becomes exponentially convergent.

The Green tensor becomes particularly simple on the cavity axis, $\rho=0$ where only a few of the functions $J_{m}(\eta \rho), J_{m}^{\prime}(\eta \rho)$ are different from zero. We have for $m=0,1,2, \ldots$

$$
\begin{aligned}
\frac{m^{2}}{\eta^{2} \rho^{2}} J_{m}^{2}(\eta \rho) & \rightarrow 0, \frac{1}{4}, 0,0, \ldots \\
J_{m}^{\prime 2}(\eta \rho) & \rightarrow 0, \frac{1}{4}, 0,0, \ldots \\
J_{m}^{2}(\eta \rho) & \rightarrow 1,0,0,0, \ldots
\end{aligned}
$$

so that

$$
\begin{aligned}
\left.\mathbf{G}^{(1)}(\mathbf{r}, \mathbf{r}, \omega)\right|_{\rho=0}=\frac{i}{8 \pi} \int_{0}^{\infty} d q\left[\frac{2 \eta^{2}}{k^{2}} r_{N}^{m=0} \hat{\mathbf{z}} \hat{\mathbf{z}}\right. \\
\left.+\left(r_{M}^{m=1}+\frac{q^{2}}{k^{2}} r_{N}^{m=1}\right)(\hat{\varrho} \hat{\varrho}+\hat{\varphi} \hat{\varphi})\right] .
\end{aligned}
$$

and

$$
\begin{aligned}
\operatorname{Tr} \mathbf{G}^{(1)}(\mathbf{r}, \mathbf{r}, \omega)_{\rho=0}=\frac{i}{4 \pi} \int_{0}^{\infty} d q\left(\frac{\eta^{2}}{k^{2}} r_{N}^{m=0}\right. \\
\left.+r_{M}^{m=1}+\frac{q^{2}}{k^{2}} r_{N}^{m=1}\right) .
\end{aligned}
$$

Finally, let us briefly discuss the Green tensor at purely imaginary frequencies $\omega=i \xi$ as required for the nonresonant potentials (2.3) and (2.5). We have $\eta=i \sqrt{\xi^{2} / c^{2}+q^{2}} \equiv i \zeta$, so the arguments of the cylindrical Bessel and Hankel functions appearing in the reflection coefficients (2.15)-(2.17) become purely imaginary. One has

$$
\begin{aligned}
J_{m}(i y) & =i^{m} I_{m}(y), \\
J_{m}^{\prime}(i y) & =i^{m-1} I_{m}^{\prime}(y), \\
H_{m}^{(1)}(i y) & =\frac{2}{\pi} i^{-(m+1)} K_{m}(y), \\
H_{m}^{(1) \prime}(i y) & =\frac{2}{\pi} i^{-m} K_{m}^{\prime}(y),
\end{aligned}
$$

hence the reflection coefficients become $(\sigma=M, N)$

$$
r_{\sigma}(i \xi)=\frac{2 i}{\pi}(-1)^{m} \frac{K_{m}(y)}{I_{m}(y)} \tilde{r}_{\sigma}(i \xi)
$$

The reduced reflection coefficients are found from (2.16) with the substitutions $x \rightarrow i y=i \zeta R, x_{1} \rightarrow i y_{1}=i \zeta_{1} R$ with $\zeta_{1}=R \sqrt{\varepsilon(i \xi) \xi^{2} / c^{2}+q^{2}}$, and $\tilde{\jmath} \rightarrow \tilde{\imath} / i, \tilde{h} \rightarrow \tilde{k} / i$ with reduced modified Bessel functions analogous to (2.19):

$$
\tilde{\imath}(y)=\frac{I_{n}^{\prime}(y)}{I_{n}(y)} ; \quad \tilde{k}(y)=\frac{K_{n}^{\prime}(y)}{K_{n}(y)} .
$$

Using the reflection coefficients (2.26), the Green tensor (2.20) at purely imaginary frequencies is seen to be purely real and given by $(\kappa=\xi / c)$

$$
\begin{aligned}
\mathbf{G}^{(1)}(\mathbf{r}, \mathbf{r}, i \xi)=\frac{1}{\pi^{2}} \int_{0}^{\infty} d q \sum_{m=0}^{\infty} \frac{K_{m}(\zeta \rho)}{I_{m}(\zeta \rho)} \\
\times\left\{\left[\frac{m^{2}}{\zeta^{2} \rho^{2}} I_{m}^{2}(\zeta \rho) \tilde{r}_{M}-\frac{q^{2}}{\kappa^{2}} I_{m}^{\prime 2}(\zeta \rho) \tilde{r}_{N}\right] \hat{\varrho} \hat{\varrho}\right. \\
+\left[I_{m}^{\prime 2}(\eta \rho) \tilde{r}_{M}-\frac{m^{2} q^{2}}{\kappa^{2} \zeta^{2} \rho^{2}} I_{m}^{2}(\zeta \rho) \tilde{r}_{N}\right] \hat{\boldsymbol{\varphi}} \hat{\boldsymbol{\varphi}} \\
\left.-\frac{\zeta^{2}}{\kappa^{2}} I_{m}^{2}(\zeta \rho) \tilde{r}_{N} \hat{\mathbf{z}} \hat{\mathbf{z}}\right\} .
\end{aligned}
$$


Correspondingly, its trace reads

$$
\begin{gathered}
\operatorname{Tr} \mathbf{G}^{(1)}(\rho, \rho, i \xi)=\frac{1}{\pi^{2}} \int_{0}^{\infty} d q \sum_{m=0}^{\infty},\left\{\left(\tilde{r}_{M}-\frac{\zeta^{2}}{\kappa^{2}} \tilde{r}_{N}\right)\right. \\
\left.\times\left[\frac{m^{2}}{\zeta^{2} \rho^{2}} I_{m}^{2}(\zeta \rho)+I_{m}^{\prime 2}(\zeta \rho)\right]-\frac{\zeta^{2}}{\kappa^{2}} \tilde{r}_{N} I_{m}^{2}(\zeta \rho)\right\} \frac{K_{m}(\zeta \rho)}{I_{m}(\zeta \rho)} .
\end{gathered}
$$

Note that for purely imaginary frequencies, the $q$-integration in the Green tensor is unproblematic as $\eta$ remains finite along the real $q$-axis.

\section{RESONANT RADII}

Combining the general expressions for the CP potential given in Sec. II A with the Green tensor of the cylindrical cavity as laid out in Sec. II C, we can explicitly calculate the full potential of any particular atom or molecule in a given state placed in a cavity of given size and material. Such examples, which require a numerical analysis, will be given in Sec. V.

Our main intention is to enhance the resonant potential by means of the cavity. To that end, it is worth recalling the case of planar cavity: The resonant CP potential of an atom placed between two plane surfaces is enhanced for certain interplate separations [23]. For a given transition frequency there exists a series of such resonant separations corresponding to integer multiples of the atomic transition wavelength. In such a case, the transition is (near-)resonant with a standing-wave mode of the planar cavity. Mathematically speaking, the enhancement results from a closest matching of the transition frequency with a pole of the scattering Green tensor.

Similarly, for the cylindrical cavity we find that for a given transition frequency there exists a series of discrete radii such that the transition is near-resonant with one of the cavity modes. In this section, we explore analytically the structure of these resonances.

\section{A. Perfect reflector}

Let us begin with the limit $|\varepsilon| \rightarrow \infty$ of a perfectly conducting cavity. For large $|\varepsilon|$, the leading order terms of the coefficients $A$ and $B_{M, N, D}$ in Eqs. (2.17) read

$$
\begin{aligned}
A & \sim-m^{2}(k R)^{2}(q R)^{2} \varepsilon^{2}+\mathcal{O}(\varepsilon) \\
B_{M} & \sim-(k R)^{3}(\eta R)^{3} \tilde{h}_{1} \tilde{h} \varepsilon^{5 / 2}+\mathcal{O}\left(\varepsilon^{2}\right) \\
B_{N} \sim B_{D} & \sim-(k R)^{3}(\eta R)^{3} \tilde{h}_{1} \tilde{\jmath} \varepsilon^{5 / 2}+\mathcal{O}\left(\varepsilon^{2}\right) .
\end{aligned}
$$

Hence, in the limit $|\varepsilon| \rightarrow \infty$, Eq. (2.16) simplifies to

$$
\tilde{r}_{M} \stackrel{|\varepsilon| \rightarrow \infty}{\longrightarrow} \frac{\tilde{h}}{\tilde{j}} ; \quad \tilde{r}_{N} \stackrel{|\varepsilon| \rightarrow \infty}{\longrightarrow} 1,
$$

and so the reflection coefficients (2.15) become

$$
r_{M} \stackrel{|\varepsilon| \rightarrow \infty}{\longrightarrow}-\frac{H_{m}^{(1) \prime}(x)}{J_{m}^{\prime}(x)} ; \quad r_{N} \stackrel{|\varepsilon| \rightarrow \infty}{\longrightarrow}-\frac{H_{m}^{(1)}(x)}{J_{m}(x)} .
$$

The potential (2.2) diverges, and is thus truly resonant, when at least one of these coefficients has a pole at $q=0$. Since $x=\eta R=k R$ for $h=0$, it is clear that resonances occur in the perfectly reflecting limit when either $J_{m}(k R)=0$ or $J_{m}^{\prime}(k R)=0$, that is, when the radius equals one of the radii given as

$$
R_{m j}^{(\prime)}=\frac{c}{\omega} j_{m j}^{(\prime)}
$$

where $j_{m j}$ and $j_{m j}^{\prime}$ are the $j$ th zero of $J_{m}(x)$ and $J_{m}^{\prime}(x)$, respectively (only zeros $j_{m j}>0$ are considered).

For each mode $m$ and each polarisation, there is hence a number of possible radii $R_{m j}$ leading to a resonant enhancement of the CP potential. The strongest resonance is that corresponding to the smallest resonant radius, which is $R_{11}^{\prime}$, corresponding to the first zero of $J_{1}^{\prime}$, $j_{11}^{\prime} \approx 1.8411838$. If the dominant transition $|n\rangle \rightarrow|k\rangle$ is a downward one, i.e. $\omega_{k n}<0$, this resonance corresponds to a potential minimum which can act as a guiding potential. For an upward transition (such as will be the case e.g. for a ground state molecule), the strongest resonance which corresponds to a potential minimum in the cylinder center is $R=R_{11}=R_{01}^{\prime}$, which happens to be a double resonance since $j_{11}=j_{01}^{\prime} \approx 3.8317060$. We will analyse these examples further in section $\mathrm{V}$.

\section{B. Good conductor}

In reality, any metal has a finite conductivity so that $|\varepsilon|<\infty$. As we will now show, this results in a shifting of the values of $k R$ which give poles at $q=0$ away from the zeros of the Bessel functions. As a consequence, the optimal radii for enhancing the $\mathrm{CP}$ potential or transition rates deviate from their perfect-conductor values as given by Eq. (3.4). We derive approximate formulae for the new optimal radii, valid for good conductors.

In the following we consider a metal described by the Drude model,

$$
\varepsilon(\omega)=1-\frac{\omega_{\mathrm{p}}^{2}}{\omega(\omega+i \gamma)}=1-\frac{\omega_{\mathrm{p}}^{2}}{\omega^{2}+\gamma^{2}}+i \frac{\gamma}{\omega} \frac{\omega_{\mathrm{p}}^{2}}{\omega^{2}+\gamma^{2}}
$$

where $\omega_{\mathrm{p}}$ is the plasma frequency and $\gamma$ is the relaxation frequency. For good conductors one has $\omega_{\mathrm{p}} \gg \gamma$, and we assume this is true in the following. Since we are considering molecules, we will restrict our interest to low frequencies and therefore also assume $\omega \ll \omega_{\mathrm{p}}$. Under these assumptions the following is true

$$
\operatorname{Re} \varepsilon<0 ; \quad \operatorname{Im} \varepsilon>0 ; \quad|\operatorname{Re} \varepsilon|, \operatorname{Im} \varepsilon \gg 1 .
$$

Let us consider the reflection coefficients $r_{M, N}$ as given by Eqs. (2.15)-(2.17) once more. We are looking for the 
complex resonant value of $k R$ which corresponds to a zero of the denominator of $r_{M, N}$ when $q=0$. A vanishes quadratically for small $q$, so we can set $A=0$ in the following and consider the coefficients $B_{M, N, D}$. The sought value of $k R$ is hence the solution of the equation $\left.B_{D}\right|_{q=0}=0$ [42], which may be written as

$$
\frac{\tilde{h}(\sqrt{\varepsilon} k R)}{\tilde{\jmath}(k R)}+\frac{\tilde{\jmath}(k R)}{\tilde{h}(\sqrt{\varepsilon} k R)}=\sqrt{\varepsilon}+\frac{1}{\sqrt{\varepsilon}} .
$$

The two roots of this second-order equation in $\tilde{\jmath}(k R)$ give all the resonances since $B_{D}$ is the denomiator of both reduced reflection coefficients for $q=0$. The perfect conductor limit $|\varepsilon| \rightarrow \infty$ is easily recovered from this equation, in which case the solutions $\tilde{\jmath}(k R)=\infty$ and $\tilde{\jmath}(k R)=0$ are just the solutions (3.4), i.e., $k R=j_{m n}$ and $k R=j_{m n}^{\prime}$.

Approximate solutions to (3.7) when $|\varepsilon|$ is large but finite are straightforward to find. We write

$$
k R=j_{m j}^{(\prime)}+\delta^{(\prime)}
$$

where $\delta^{(\prime)}$ are small complex numbers. The prime corresponds to solutions close to a zero of $J_{m}^{\prime}$. Solving Eq. (3.7) to leading order in $\varepsilon^{-1}$ then gives

$$
\begin{gathered}
\delta \approx-\frac{i}{\sqrt{\varepsilon}} ; \\
\delta^{\prime}=\delta_{m j}^{\prime} \approx \frac{J_{m}\left(j_{m j}^{\prime}\right)}{J_{m}^{\prime \prime}\left(j_{m j}^{\prime}\right)} \frac{i}{\sqrt{\varepsilon}} ;
\end{gathered}
$$

note that $\tilde{h}(z) \sim i+\mathcal{O}\left(z^{-1}\right), \quad|z| \rightarrow \infty$ (cf. e.g. [40] $\S 9.2)$. The fraction $J_{m}\left(j_{m j}^{\prime}\right) / J_{m}^{\prime \prime}\left(j_{m j}^{\prime}\right)$ is a real and negative number of order unity which tends asymptotically to -1 for large arguments. For a good conductor (3.6), $\varepsilon$ is in the second quadrant of the complex plane, so $\sqrt{\varepsilon}$ is in the first quadrant for an absorbing medium, and $i / \sqrt{\varepsilon}$ is in the first complex quadrant. The shifts $\delta$ and $\delta^{\prime}$ to the poles at $q=0$ lie in the third quadrant.

Similarly, one can show that all poles of the integrand of the scattering Green tensor of a well conducting surface are displaced from the Bessel zeros $\eta R=j_{m j}$ and $\eta R=$ $j_{m j}^{\prime}$ by small quantities which lie in the third complex quadrant. This means that the poles of the reflection coefficients all lie in the lower half of the complex $\eta$ plane when $|\varepsilon|<\infty$, justifying the rotation of $q$-integral path mentioned in Sec. II C and shown in Fig. 2.

For real frequencies, the cavity radius can never be chosen such that it lies exactly on one of the complex-valued resonances. Instead, we will derive optimal real radii close to the resonances that maximise the real or imaginary parts of the Green tensor. As these optimal radii turn out to be different for the real vs imaginary parts, we have to distinguish between radii which maximise the resonant potential (2.7) and those which maximise the heating rate $(2.11 \mathrm{~b})$.

\section{Optimal radii for enhancing the potential}

Consider first a resonance associated with a pole of $r_{N}$. We represent the pole in the form (3.8), and let $q$ and $\delta$ be small, so that

$$
x=\eta R \approx j_{n j}+\delta-\frac{1}{2} j_{n j} \frac{q^{2}}{k^{2}} .
$$

Keeping only leading orders in the small quantities $\delta, q^{2}$ and $1 / \sqrt{\varepsilon}$, i.e., using Eqs. (3.1a)-(3.1c) with

$$
\begin{aligned}
B_{N} \sim & -(k R)^{3}(\eta R)^{3} \tilde{h}_{1} \tilde{\jmath}^{5 / 2}+(k R)^{4}(\eta R)^{2} \tilde{\jmath}^{2} \varepsilon^{2} \\
& +\mathcal{O}\left(\varepsilon^{3 / 2}\right),
\end{aligned}
$$

the reflection coefficient as given by (2.15)-(2.17) becomes

$$
\begin{aligned}
r_{N} & \approx \frac{i \sqrt{\varepsilon} H_{m}^{(1)}(x)}{i \sqrt{\varepsilon} J_{m}(x)-J_{m}^{\prime}(x)} \\
& \approx-\frac{i Y_{m}\left(j_{m j}\right)}{J_{m}^{\prime}\left(j_{m j}\right)} \frac{1}{q^{2} j_{m j} /\left(2 k^{2}\right)-\zeta}
\end{aligned}
$$

with

$$
\zeta=\delta+i / \sqrt{\varepsilon}
$$

We have used that $\tilde{h}_{1} \approx i$ because of its argument being large with positive imaginary part (see the asymptotic expansions, section 9.2 of [40]) and noted that $H_{m}^{(1)}(x) \approx$ $i Y_{n}(x)$.

Now we note that in the integrand of the integral (2.20), the term which resonates is the last one, which does not have a prefactor $q^{2}$. For this term there is no other resonating structures in the integrand, and we can simply conclude that close to a strong resonance

$$
\begin{aligned}
U_{n}^{\mathrm{res}}(\mathbf{r}) & \propto \operatorname{Re} \mathbf{G}(\mathbf{r}, \mathbf{r}, \omega) \propto \operatorname{Im} \int_{0}^{\infty} d q r_{N} \\
& \propto \operatorname{Re} \int_{0}^{\infty} \frac{d q}{q^{2}-\zeta} \propto \operatorname{Re} \sqrt{\frac{1}{-\zeta}} \propto \operatorname{Im} \sqrt{\frac{1}{\zeta}},
\end{aligned}
$$

where we have made the substitution $q \rightarrow \sqrt{2 / j_{m j}} q / k$ at the beginning of the second line. Explicitly, we have

$$
\begin{gathered}
\operatorname{Im} \sqrt{\frac{1}{\zeta}}=-|\zeta|^{-1 / 2} \sin \left[\frac{1}{2} \arctan \frac{\operatorname{Im} \zeta}{\operatorname{Re} \zeta}\right] \\
=\frac{-1}{\sqrt{2} \sqrt[4]{\operatorname{Re}^{2} \zeta+\operatorname{Im}^{2} \zeta}} \sqrt{1-\frac{\operatorname{Re} \zeta}{\sqrt{\operatorname{Re}^{2} \zeta+\operatorname{Im}^{2} \zeta}}} .
\end{gathered}
$$

where $\operatorname{Re} \zeta=\delta-\operatorname{Im}\left\{\varepsilon^{-1 / 2}\right\}$ and $\operatorname{Im} \zeta=\operatorname{Re}\left\{\varepsilon^{-1 / 2}\right\}$. Differentiating this result with respect to $\delta$, we find that the maximum lies at

$$
\operatorname{Re} \zeta=-\operatorname{Im} \zeta / \sqrt{3},
$$




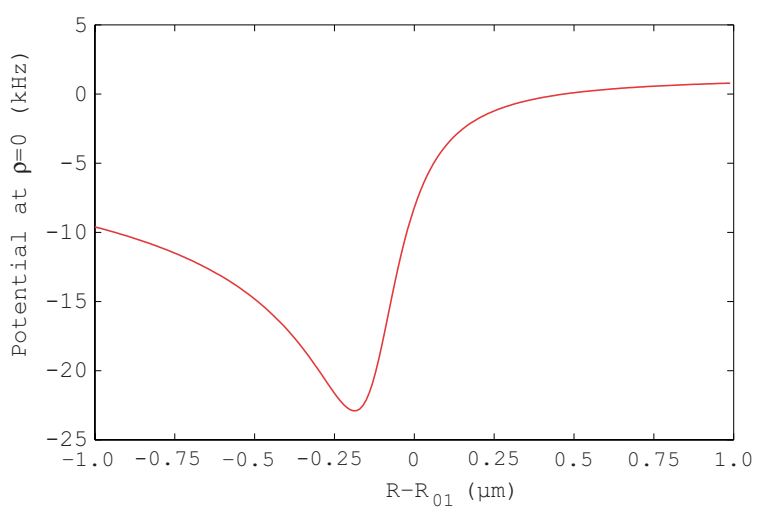

FIG. 3: The potential of Rydberg Rb in its 32s state at the centre $(\rho=0)$ of a Au cavity, as a function of radius close to the resonance corresponding to the first zero $j_{01} \approx 2.4$ of $J_{0}$. The cavity resonantly enhances the contributino from the $32 \mathrm{~s}_{1 / 2} \rightarrow 31 \mathrm{p}_{3 / 2}$ transition. $R_{01}=799.9 \mu \mathrm{m}$ as given by Eq. (3.4) is the corresponding perfect conductor resonant radius.

so that

$$
\delta \approx \operatorname{Im}\left\{\varepsilon^{-1 / 2}\right\}-\frac{1}{\sqrt{3}} \operatorname{Re}\left\{\varepsilon^{-1 / 2}\right\}
$$

Note that $\delta$ is independent of $m$ and $j$ to leading order in the large parameter $\varepsilon$. The resonant radii associated with resonances of $r_{N}$ are hence given by

$$
k R \approx j_{m j}+\operatorname{Im}\left\{\varepsilon^{-1 / 2}\right\}-\frac{1}{\sqrt{3}} \operatorname{Re}\left\{\varepsilon^{-1 / 2}\right\} .
$$

For resonances due to $r_{M}$, we present the poles of $J_{m}^{\prime}(x)$ in the form (3.8). A virtually identical procedure then leads to

$$
\delta^{\prime}=\delta_{m j}^{\prime} \approx-\frac{J_{m}\left(j_{m j}^{\prime}\right)}{J_{m}^{\prime \prime}\left(j_{m j}^{\prime}\right)} \delta
$$

Consequently, the respective resonant radii read

$$
k R \approx j_{m j}-\frac{J_{m}\left(j_{m j}^{\prime}\right)}{J_{m}^{\prime \prime}\left(j_{m j}^{\prime}\right)}\left(\operatorname{Im}\left\{\varepsilon^{-1 / 2}\right\}-\frac{1}{\sqrt{3}} \operatorname{Re}\left\{\varepsilon^{-1 / 2}\right\}\right) .
$$

As a numerical example, take $\mathrm{Rb}$ in its 32s Rydberg state whose dominating $|n\rangle=32 \mathrm{~s}_{1 / 2} \rightarrow|k\rangle=31 \mathrm{p}_{3 / 2}$ transition has frequency $\omega_{k n} \approx-9.013 \times 10^{11} \mathrm{rad} / \mathrm{s}$ and a cylinder made of Au using $\omega_{\mathrm{p}} \approx 1.4 \times 10^{16}, \gamma \approx 5.4 \times 10^{13}$ [35]. In this case, the shifts of the maxima away from the Bessel zero for for resonances due to $r_{N}$ are found to be $\delta \approx-0.00056$.

In Fig. 3, we show the potential as a function of radius close to the smallest resonance at $k R_{01} \approx j_{01}$, resonating with the smallest downward transition to $31 \mathrm{p}$. More details on the specifics of Casimir-Polder potentials on Rydberg atoms are found in Ref. [25] and summarised in Sec. V below. It is interesting to note that even though gold is a good conductor whose permittivity is much greater than unity as assumed above, the shift of the resonant radius away from the perfect-condutor result is not negligible. We see in Fig. 3 that the potential at the optimal radius is about a factor 2.5 greater than its value for $k R_{01}=j_{01}$. The resonant radius is given with excellent approximation by Eq. (3.17) which for this example predicts the maximum at $R-R_{01} \approx-187 \mathrm{~nm}$.

We note furthermore that the width of the radius resonances is in the order of $500 \mathrm{~nm}$, which is expected to be well within the accuracy obtainable for production of pipes with diameters in the order of hundreds of microns. It is also much wider than surface roughness amplitudes of good metal surfaces, indicating that the associated diminishing of the CP-potential enhancement is not expected to be important. The narrowness of the peaks are thus in the order of one three thousandth of the cylinder radius, and we do not expect observation and utilisation of the resonant behaviour to be hampered by issues of production accuracy.

\section{Optimal radii for enhancing transition rates}

Optimal radii for resonantly enhancing transition rates in a conducting cavity can be derived in close analogy to the previous section. We again start with resonances of $r_{N}$ as approximated by Eq. (3.11). The transition rates $(2.11 \mathrm{~b})$ close to a resonance are found just as in (3.13) to be proportional to

$$
\Gamma_{n}^{(1)}(\mathbf{r}) \propto \operatorname{Im} \mathbf{G}(\mathbf{r}, \mathbf{r}, \omega) \propto \operatorname{Re} \sqrt{\frac{1}{\zeta}}
$$

where

$$
\begin{aligned}
\operatorname{Re} & \sqrt{\frac{1}{\zeta}}=|\zeta|^{-1 / 2} \cos \left[\frac{1}{2} \arctan \frac{\operatorname{Im} \zeta}{\operatorname{Re} \zeta}\right] \\
= & \frac{1}{\sqrt{2} \sqrt[4]{\operatorname{Re}^{2} \zeta+\operatorname{Im}^{2} \zeta}} \sqrt{1+\frac{\operatorname{Re} \zeta}{\sqrt{\operatorname{Re}^{2} \zeta+\operatorname{Im}^{2} \zeta}}}
\end{aligned}
$$

The maximum of the above function is again found by differentiation w.r.t. $\delta$. It now lies at $\operatorname{Re} \zeta=\operatorname{Im} \zeta / \sqrt{3}$, so that

$$
\delta \approx \operatorname{Im}\left\{\varepsilon^{-1 / 2}\right\}+\frac{1}{\sqrt{3}} \operatorname{Re}\left\{\varepsilon^{-1 / 2}\right\}
$$

is again independent of $m$ and $j$ to leading order in $\varepsilon$. The resonant radii associated with resonances of $r_{N}$ for enhancing transition rates

$$
k R \approx j_{m j}+\operatorname{Im}\left\{\varepsilon^{-1 / 2}\right\}+\frac{1}{\sqrt{3}} \operatorname{Re}\left\{\varepsilon^{-1 / 2}\right\}
$$

are thus different from the corresponding radii (3.17) for enhancing the $\mathrm{CP}$ potential.

As for the potential, the resonances due to $r_{M}$ are found to be maximal at

$$
\delta_{m j}^{\prime} \approx-\frac{J_{m}\left(j_{m j}^{\prime}\right)}{J_{m}^{\prime \prime}\left(j_{m j}^{\prime}\right)} \delta
$$




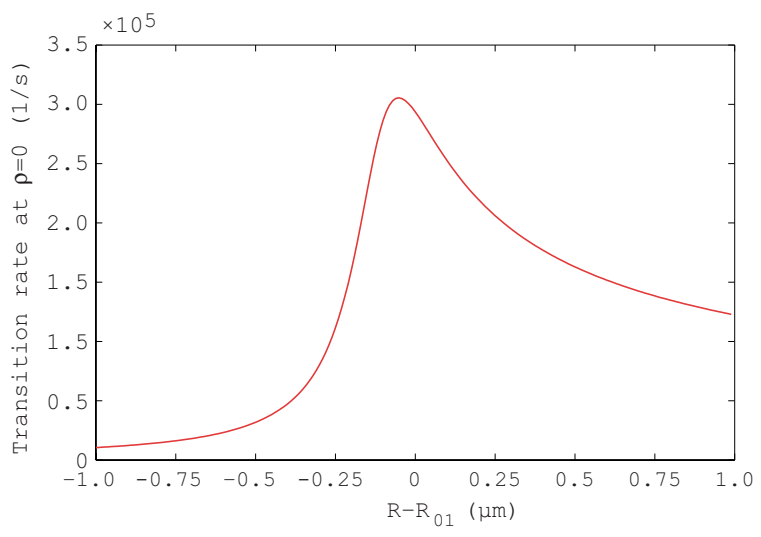

FIG. 4: Same set-up as Fig. 3, but for the cavity-assisted transition rate from state $32 \mathrm{~s}$ to $32 \mathrm{p}_{3 / 2}$.

$$
\begin{aligned}
& \text { i.e., } \\
& \qquad k R \approx j_{m j}-\frac{J_{m}\left(j_{m j}^{\prime}\right)}{J_{m}^{\prime \prime}\left(j_{m j}^{\prime}\right)}\left(\operatorname{Im}\left\{\varepsilon^{-1 / 2}\right\}+\frac{1}{\sqrt{3}} \operatorname{Re}\left\{\varepsilon^{-1 / 2}\right\}\right) .
\end{aligned}
$$

The resonant enhancement of the total transition rate out of the $32 \mathrm{~s}$ state of Rydberg $\mathrm{Rb}$ placed at the centre of a $\mathrm{Au}$ cavity is shown in Fig. 4, again for the $j_{01}$ resonance of $r_{N}$. The true optinal radius is seen to be well aproximated by Eq. (3.23), it is smaller than the perfect-conductor value by $0.004 \%$. The optimal radii for enhancing potential vs rate thus differ noteably by about $192 \mathrm{~nm}$.

\section{GENERAL SCALING PROPERTIES}

In this section we disucss the scaling properties of resonant thermal $\mathrm{CP}$ forces, i.e., their dependences on the relevant molecular, material, thermal and geometric parameters. These were discussed in detail for the case of a planar cavity in Ref. [23]; and many of the results remain valid also in the cylindrical geometry: The resonant potential corresponding to a dipole transition from state $n$ to state $k$ is proportional to the absolute square of the transition dipole moment, $\left|\mathbf{d}_{k n}\right|^{2}$. Likewise, the resonant potential scales with temperature just as in the planar case; proportional to the photon number $n\left(\left|\omega_{k n}\right|\right)$. For temperatures larger than the transition frequency the temperature dependence is thus approximately linear, $U^{\text {res }}(\rho) \propto T, k_{\mathrm{B}} T \gg \hbar \omega_{k n}$. In the opposite limit the resonant potential is exponentially suppressed: $U^{\mathrm{res}}(\rho) \propto \exp \left(-\hbar \omega_{k n} / k_{\mathrm{B}} T\right), k_{\mathrm{B}} T \ll \hbar \omega_{k n}$.

On the contrary, the scaling of the potential with the permittivity of the cavity walls depends strongly on the geometry. For the cylindrical cavity, this scaling follows immediately from the discussion of the resonant radii in Sec. III C. As shown by Eq. (3.13), the resonant potential is proportional to $\sqrt{1 / \zeta}$. This function (3.14) has its maximum at $\operatorname{Re} \zeta=-\operatorname{Im} \zeta / \sqrt{3}$, where it takes the value

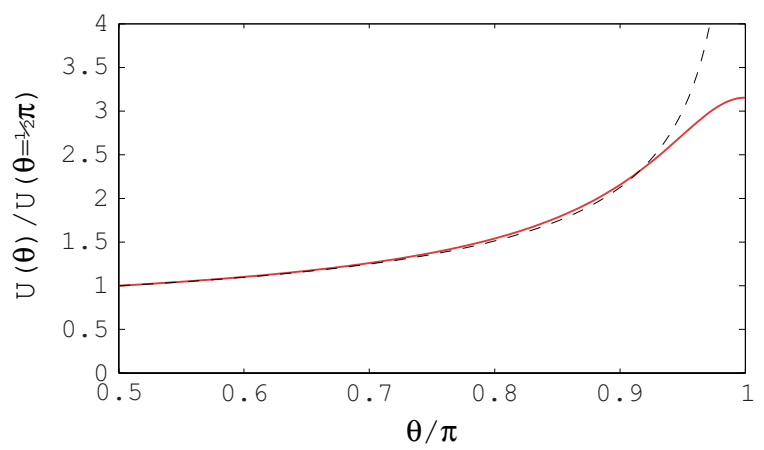

FIG. 5: The dependence of the peak potential at resonance on $\theta=\arg (\varepsilon)$. The solid graph is $U^{\text {res }}$ calculated for a $32 \mathrm{~s}$ Rydberg atom whose $32 \mathrm{~s} \rightarrow 31 \mathrm{p}$ transition is in resonance with the $j_{01}$ zero of $J_{0}(x)$, varying the phase of $\varepsilon$ throughout the physical range but keeping $|\varepsilon|$ constant and equal to its value using Eq. (3.5). The dashed graph is the theoretical curve based on the approximate scaling of Eq. (4.1). Both functions have been normalized by their values at $\arg \varepsilon=\pi / 2$.

$\sqrt[4]{3} /(2 \sqrt{2 \operatorname{Im} \zeta})$ with $\operatorname{Im} \zeta=\operatorname{Re}\left\{\varepsilon^{-1 / 2}\right\}$. To leading order in the large quantity $\varepsilon$, the potential for near-resonant radius hence scales as

$$
U_{n}^{\mathrm{res}}(\mathbf{r}) \propto \frac{|\varepsilon|^{1 / 4}}{\sqrt{\cos \left[\frac{1}{2} \arg (\varepsilon)\right]}} .
$$

Note that this scaling is an improvement with respect to the enhancement achievable in a planar cavity for which we found the potential to be proportional to $\ln \varepsilon$ [23]. The resonant enhancement in the above approximation diverges as $\arg (\varepsilon) \rightarrow \pi$. A full numerical investigation shows that the potential in fact remains finite in this limit, cf. Fig. 5. In the displayed example for the $\arg (\varepsilon)$ dependence of the potential of Rydberg $\mathrm{Rb}$ in its $32 \mathrm{~s}$ state, the value of the potential changes by more than a factor of three as the phase of $\varepsilon$ is varied from $\pi / 2$ (purely imaginary permittivity) to $\pi$ (purely real permittivity). This implies that decreasing the dissipation rate of the cavity material can increase the enhancement significantly. Note that with $\gamma \approx 5.4 \times 10^{13}$ [35], the actual phase of $\mathrm{Au}$ at the relevant transition frequency is close to $\pi / 2$.

The shown dependence of the resonant potential on the phase of the permittivity is also interesting in light of the thermal anomaly of the Casimir effect for metals $[36,37]$. This dispute centers in an essential way on the description of the dissipation of the metal: employing the standard Drude model (3.5) with measured optical dissipation data gives a different prediction of the force at high temperature than that using a non-dissipative plasma model in which one sets $\gamma=0$ at the outset. Experiments appear to favour the latter approach [37, 38]. The cavity enhancement of the $\mathrm{CP}$ potential as a related quantum vacuum effect is good system for investigating the thermal anomaly further: As Eq. (4.1) shows, the potential at resonance using a plasma model predicts a sig- 
nal more than three times that calculated for the Drude model (note that $|\varepsilon|$ is also larger using a plasma model, adding to the relative difference in prediction).

Finally, let us consider the scaling of the potential with the transition frequency. The frequency infuences the potential (2.7) in three ways: Firstly, there is a prefactor $\omega_{k n}^{2} n\left(\left|\omega_{k n}\right|\right)$, independently of the particular cavity geometry considered. Secondly, there is an additional factor of $\omega_{n k}$ because the magnitude of oscillations fall of as $J_{m}^{2}, J_{m}^{\prime 2} \sim 1 /(R-\rho)$ away from the cavity walls and the resonant cavity radius is in turn proportional to $\omega_{k n}$. This geometric frequency-dependence is closely similar to that found for a planar cavity [23]; it is a consequence of the general scaling law for the Green tensor as established in Ref. [39]. Thirdly, the $\varepsilon$-scaling introduces an additional frequency dependence. For $\omega_{k n} \ll \gamma$, Eq. (4.1) leads to a $\sqrt[4]{1 / \omega_{k n}}$ scaling. Combining these three effects, the peak resonant potentials scale as $\omega_{k n}^{2.75} n\left(\left|\omega_{k n}\right|\right)$. For comparison, the scaling $\omega_{k n}^{3} n\left(\left|\omega_{k n}\right|\right)$ for a planar cavity is slightly stronger due to the much weaker $\varepsilon$ dependence.

For atoms in highly excited Rydberg states (cf. Sec. V below), the CP potential is dominated by transitions to neighbouring states. For these transitions, the frequencies and dipole matrix elements depend in a simple way on the principal quantum number $n$ of the initial atomic Rydberg state: For sufficienty large $n$, the transition frequencies can be given as $\omega_{k n}=2 \mathrm{Ry} /\left(\hbar n^{3}\right)$ (Ry, Rydberg energy) while the dipole moments scale as $n^{2}$. Combining this with the dependencies discussed above, one finds that the CP potential of a Rydberg atom scales as

$$
\begin{aligned}
U_{n}^{\text {res }} & \propto n^{-4.25} n\left(\omega_{k n}\right) \\
& \propto\left\{\begin{array}{cl}
n^{-1.25}, & n \gg n_{T} ; \\
\exp \left[-\left(n_{T} / n\right)^{3}\right] & n \ll n_{T},
\end{array}\right.
\end{aligned}
$$

where we have introduced a characteristic thermal principal quantum number

$$
n_{T}=\left(\frac{2 \mathrm{Ry}}{k_{\mathrm{B}} T}\right)^{\frac{1}{3}}
$$

The maximum potential is found for states with a principal quantum number around $n \sim n_{T}$. At room temperature, $n_{T} \approx 10.2$, hence the maximum potential is found for principal quantum numbers below the Rydberg range. For this reason we have chosen a low Rydberg state, 32s, for our numerical examples above and in the following.

\section{NUMERICAL RESULTS}

We are now going to present numerical studies of the $\mathrm{CP}$ potential inside a cylindrical cavity based on the exact formulas presented in Sec. II. Due to the complexity of the formulaic apparatus for the case of the cylinder, it is necessary to first ascertain the correctness of the numerical calculations. As a numerical benchmark, we verified that for positions sufficiently close to the cylinder wall $(R-\rho \ll R)$ the potential tends asymptotically to that outside a half-space (c.f. e.g. [13]).

We are interested in an observable resonant enhancement of the potential predicted for the radii derived in Sec. III. As clear from the previous Sec. IV, this requires a cavity made of out a good conductor and an atomic system whose transition frequencies lie close to the peak of the thermal spectrum $\omega_{k n}^{11 / 4} n\left(\left|\omega_{k n}\right|\right)$ at room temperature and whose respective dipole matrix elements are large. Rydberg atoms with their enormous matrix elements and relatively small transition frequencies fulfill both of these requirements.

In practice, Rydberg states with principal quantum number $n$ in the range 30 to 50 can readily be prepared using standard Rydberg lasers. As shown at the end of Sec. IV, the optimal choice of $n$ for measuring the resonant enhancement is smaller than the standard Rydberg regime, around $n=10$ : While transition dipole moments increase with higher $n$, transition frequencies of the dominant transition decrease further away from the optimal frequency value $\sim k_{\mathrm{B}} T$, resulting together in a low optimum $n$. As a compromise, we use the value $n=32$ for our numerical examples, being a level in the lower part of the Rydberg spectrum while still being readily available with standard equipment. In all calculation the temperature was $300 \mathrm{~K}$, although in all the cases considered herein there is virtually no discernable temperature dependence (c.f. also [28]).

The resonant potential is calculated only for the dominating transition to $31 \mathrm{p}_{3 / 2}$ - transitions to higher and lower levels contribute significantly only in the nonretarded regime close to the cylinder walls which we are not interested in in the present investigation (but see [25] for details). Results are shown in Fig. 6 for the first eight resonances of the coefficient $r_{N}$ (corresponding to zeros of $J_{m}(x)$ ), and $r_{M}$ (corresponding to zeros of $J_{m}^{\prime}(x)$ ). Note how some radii resonate both with $r_{M}$ and $r_{N}$ since $J_{0}^{\prime}(x)=-J_{1}(x)$.

For the considered case of a resonantly enhanced downward transititon, we observe that a potential at the resonant radius corresponding to $j_{m j}^{(\prime)}$ has $j$ local minima. Potentials for $j_{m j}$ have minima on the cylinder axis for even $m$ and maxima for odd $m$. For the $j_{m j}^{\prime}$ resonances, the situation is reversed. The double resonances are dominated by the $j_{m j}$ contribution, so the first of the two rules applies. For a given $m$, the maximum potential depth decreases with $j$.

One might expect that smaller radii give the deeper potential minima, based on the fact that the amplitude of oscillations decrease away from a boundary (outside a planar half-space the oscillation amplitude decreases proportional to inverse distance [13]). Unlike the planar case [23], however, this is not true in general. The lowest Bessel zeros are $j_{11}^{\prime} \approx 1.8412, j_{01} \approx 2.4048, j_{21}^{\prime} \approx 3.0542$ and $j_{01}^{\prime}=j_{11} \approx 3.8317$. The two first on this list indeed correspond to the two largest potential extrema in the same order, but $j_{21}^{\prime}$ in fact represents the shallowest of 

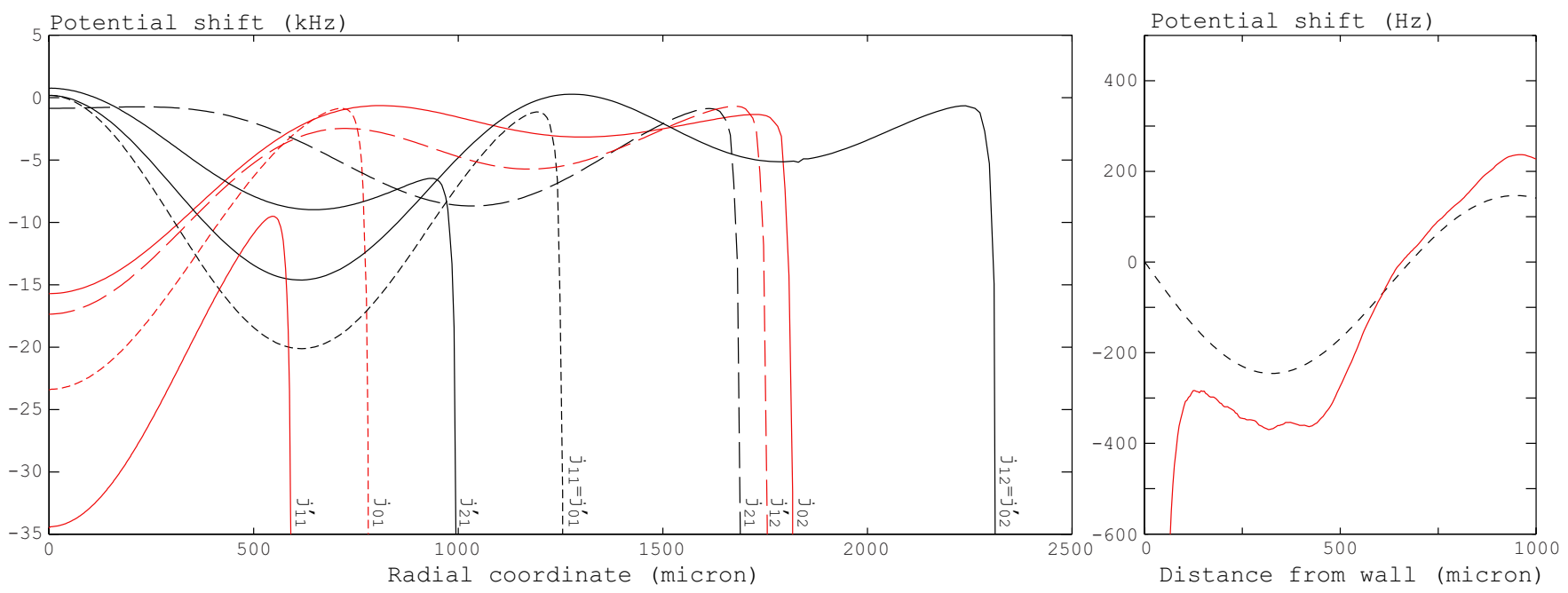

FIG. 6: (Left panel) Casimir-Polder potential at the eight smallest resonant radii acting on isotropic Rb in the state 32s, whose dominating transition to $31 \mathrm{p}_{3 / 2}$ resonantes with various modes of a gold cavity described by Eq. (3.5). The radii are chosen according to Eq. (3.17) $\left(j_{01}, j_{02}, j_{11}=j_{01}^{\prime}, j_{12}=j_{02}^{\prime}\right.$, and $\left.j_{21}\right)$ and Eq. (3.19) $\left(j_{11}^{\prime}, j_{12}^{\prime}, j_{21}^{\prime}\right)$. (Right panel) The unenhanced potential outside a plane half-space. For comparison, the oscillating part of the shift due to the transition to $31 \mathrm{p}_{3 / 2}$ alone is shown as a dotted line.
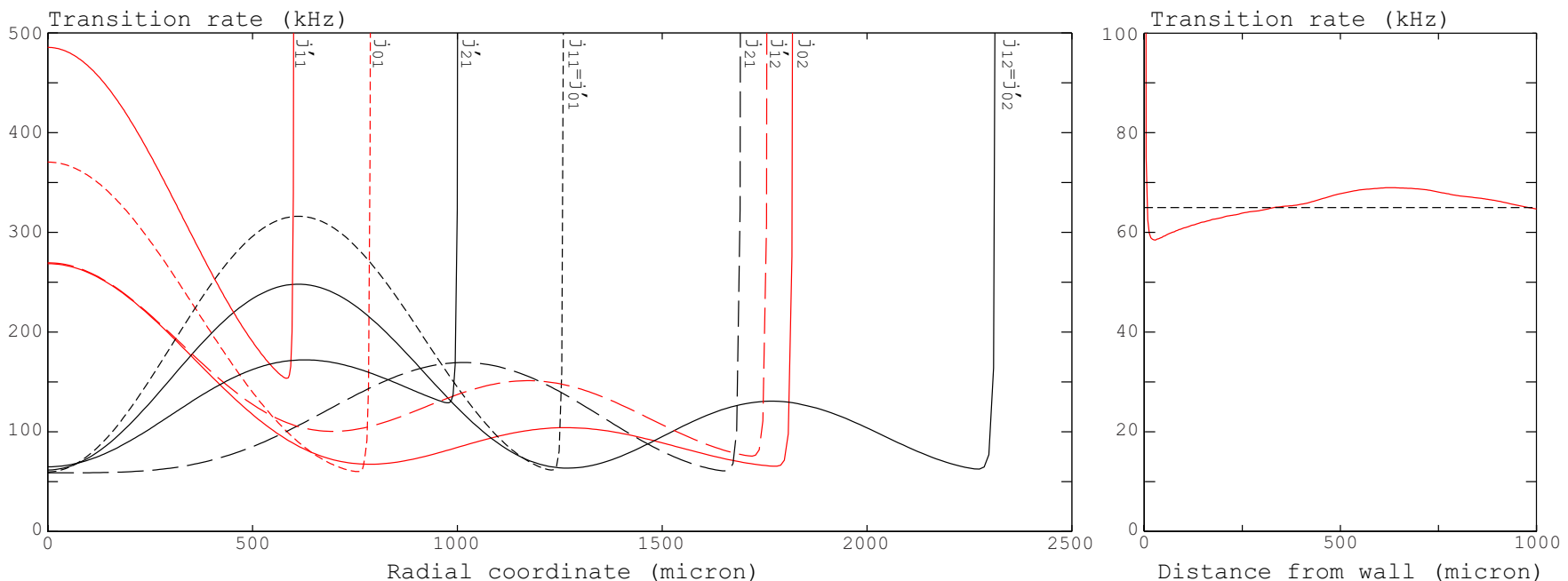

FIG. 7: (Left panel) Enhanced transition rates at the eight smallest resonant radii acting on isotropic Rb in the state 32s, whose dominating transition to $31 \mathrm{p}_{3 / 2}$ resonates with various modes of in a gold cavity described by Eq. (3.5). The radii are chosen according to Eq. (3.23) $\left(j_{01}, j_{02}, j_{11}=j_{01}^{\prime}, j_{12}=j_{02}^{\prime}\right.$, and $\left.j_{21}\right)$ and Eq. (3.25) $\left(j_{11}^{\prime}, j_{12}^{\prime}, j_{21}^{\prime}\right)$. (Right panel) The unenhanced shift outside a plane half-space. The free space transition rate is shown as a dotted line.

the eight enhanced potentials considered in Fig. 6.

The corresponding enhancement of the transition rate from 32 s to $31 \mathrm{p}_{3 / 2}$ is shown in Fig. 7 where the cylinder radii are picked according to Eq. (3.23) and Eq. (3.25) as appropriate. The figure thus shows the maximally enhanced transition rate between these two levels. The transition rate is increased by about a factor 7.4 for the smallest cavity corresponding to $j_{11}^{\prime}$ compared to the transition rate in free space, which for the $32 \mathrm{~s}$ state is about $65 \mathrm{kHz}$. In comparison, the transition rate outside a half-space oscillates about its free-space value within variations of about $10 \mathrm{kHz}$ in the far zone, hence showing that the cavity can enhance the oscillating contribution to the rate alone by about a factor 50 . The lifetime of the initial state against spontaneous and stimulated decay is thus reduced from its free-space value of $50 \mu$ s to $1.4 \mu \mathrm{s}$ inside the cylindrical cavity for the $j_{11}^{\prime}$ resonance. Recall from Sec. III that the potentials and transition rates peak at different radii. In Fig. 7, we have chosen the optimal radii for enhancing the rates. By contrast, the transition rates at the optimal radii for enhancing potentials (not displayed) are smaller by about a factor 2 . 


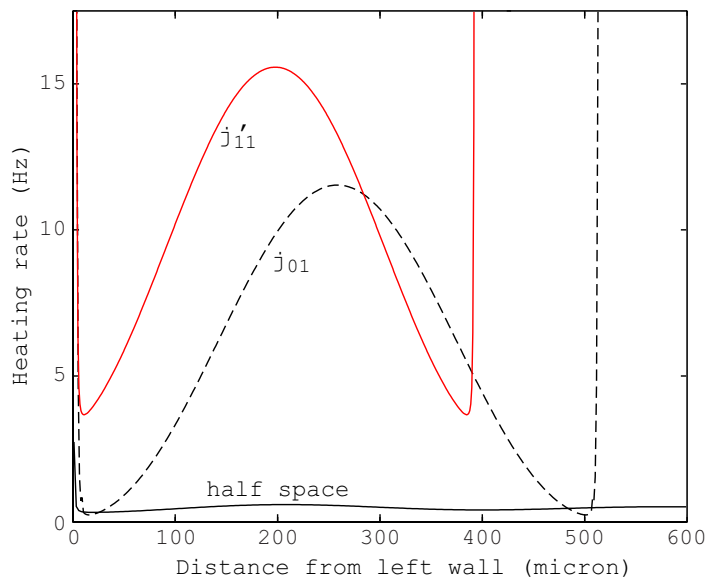

FIG. 8: Comparison of enhanced and non-enhanced heating rate of ground state $\mathrm{LiH}$. The heating rate outside a half-space [41] shown together with the two highest cylinder resonances (radii from (3.23) and (3.25)) along a central cylinder crosssection.

For comparison, we consider the case of the polar molecule LiH. Polar molecules also exhibit transitions in the frequency regime ideal for enhancement, but with the respective dipole matrix elements being much smaller than those of Rydberg atoms. $\mathrm{LiH}$ has a lowest transition frequency (rotational) of $\omega_{10}=2.79 \times 10^{12} \mathrm{rad} / \mathrm{s}$ and corresponding transition dipole moment $\left|\mathbf{d}_{10}\right|^{2}=$ $3.85 \times 10^{-58} \mathrm{C}^{2} \mathrm{~m}^{2}$. As a result of the smaller dipole moment, the $\mathrm{CP}$ potential of polar molecules is much smaller than that of Rydberg atoms. In spite of a relative enhancement of about a factor 50-100 in a cylindrical cavity, the achievable potential depth is still less than $1 \mathrm{~Hz}$, which is insufficient for moelcular guiding purposes.

While a cavity-enhancement of two orders of magnitude is modest for the CP potential of a polar molecule, it is of interest when regarding the ground-state heating rates. As Fig. 8 shows, the transition to a particular state (in this case the lowest rotational state) can be enhanced by a factor of about 30, considerably reducing the lifetime of the rotational ground-state against heating from $2.1 \mathrm{~s}$ to $0.064 \mathrm{~s}$.

\section{CONCLUSIONS}

We have presented the detailed theory for a particle (atom or molecule) in an eigenstate inside a cylindrical cavity carved out of a homogeneous and non-magnetic material. A particle out of thermal equilibrium with its environment is subject to spatially oscillating CasimirPolder forces near surfaces, and we have focused particularly on a scheme to enhance the oscillating force components by fine-tuning the cavity radius to resonate with the particle's internal transition wavelength. A similar oscillating behaviour is observed for the transition rates between eigenstates.

Formulas for calculating appropriate radii for maximal enhancement of potential and rates have been derived. For a perfectly conducting cylinder, the resonant radii are exactly given as the zeros of the Bessel function $J_{m}$ and its derivatives divided by the wave number of the resonating transition. The optimal radii are slightly shifted when the cavity is not perfectly conducting, and simple expressions for the correction have been derived for good conductors. The corrected optimal radii for enhancing potential vs transition rates are now slightly different.

We have shown how the cavity enhancement scales with the relevant paramaters of the set-up. In particular, we have paid attention to the dependence of the enhancement on the permittivity $\varepsilon\left(\omega_{k n}\right)$ of the cavity material We have shown that the potential at resonance scales as $U \propto\left|\varepsilon\left(\omega_{k n}\right)\right|^{1 / 4}$, which is a noticeable improvement over the planar geometry, for which the scaling was found to be logarithmic, $U \propto \ln \varepsilon\left(\omega_{k n}\right)$ [23]. A strong dependence is also found on the complex phase $\arg \varepsilon\left(\omega_{k n}\right)$ of the permittivity. This is interesting in light of the controversy surrounding the temperature correction to the Casimir force between metals, for which the complex phase of $\varepsilon$ for the metal in question is of the essence. A precision experiment of the potential enhancement in a cylindrical cavity could be a critical experiment in this respect.

The cases of a Rydberg atom and a ground state $\mathrm{LiH}$ molecule have been studied numerically, both of which are of experimental and technological interest. We have found that the deepest potential minima for Rydberg atoms can be obtained for quantum numbers in the lower end of the Rydberg regime. With the smallest cavity enhancement (corresponding to the first zero of $J_{1}^{\prime}(x)$ ), a guiding potential depth in excess of $30 \mathrm{kHz}$ is obtainable, which is within the region of observability of modern experiments. The enhancement factor obtained is approximately 50, an order of magnitude better than what we obtained for a planar gold cavity [23]. For the polar molecule $\mathrm{LiH}$, the cavity enhancement was found to be insufficient to bring the potential into the observable regime. Instead, a considerable enhancement of groundstate heating rates can be achieved.

\section{Acknowledgements}

We have benefited from discussions with Professor J. Fortágh. This work was supported by the UK Engineering and Physical Sciences Research Council. Support from the European Science Foundation (ESF) within the activity 'New Trends and Applications of the Casimir Effect' (www.casimir-network.com) is greatfully acknowledged. 
[1] H. B. G. Casimir and D. Polder, Phys. Rev. 73, 360 (1948).

[2] E.M. Lifshitz, Zh. Eksp. Teor. Fiz. 29, 94 (1955) [Sov. Phys. JETP 2, 73 (1956)].

[3] A.D. McLachlan, Proc. R. Soc. Lond. Ser. A 274, 80 (1963).

[4] C. Henkel, K. Joulain, J.P. Mulet, and J.-J. Greffet, J. Opt. A: Pure Appl. Opt. 4, S109 (2002).

[5] M. Antezza, L.P. Pitaevskii, and S. Stringari, Phys. Rev. Lett. 95, 113202 (2005).

[6] J.M. Obrecht, R.J. Wild, M. Antezza, L.P. Pitaevskii, S. Stringari, and E.A. Cornell, Phys. Rev. Lett. 98, 063201 (2007).

[7] T. Nakajima, P. Lambropoulos, and H. Walther, Phys. Rev. A 56, 5100 (1997).

[8] S.-T. Wu and C. Eberlein, Proc. R. Soc. Lond. Ser. A 456, 1931 (2000).

[9] S.Y. Buhmann and D.-G. Welsch, Prog. Quantum Electron. 31, 51 (2007).

[10] S. Scheel and S.Y. Buhmann, Acta Phys. Slov. 58, 675 (2008).

[11] S.Y. Buhmann and S. Scheel, Phys. Rev. Lett. 100, 253201 (2008).

[12] M.-P. Gorza and M. Ducloy, Eur. Phys. J. D 40343 (2006).

[13] S.Å. Ellingsen, S.Y. Buhmann, and S. Scheel, Phys. Rev. A 79, 052903 (2009).

[14] K.H. Drexhage, in E. Wolf (ed.) Progress in Optics XII (North-Holland, Amsterdam, 1974), p. 163.

[15] R.R. Chance, A. Prock, R. Silbey, in I. Prigogine and S.A. Rice (eds.) Advances in Chemical Physics XXXVII p.1 (1978).

[16] G. Barton, Proc. R. Soc. Lond. Ser. A 320, 251 (1970).

[17] G. Barton, Proc. R. Soc. Lond. Ser. A 367, 117, (1979).

[18] G. Barton, Proc. R. Soc. Lond. Ser. A 410, 141, (1987).

[19] W. Jhe, Phys. Rev. A 43, 5795 (1991).

[20] W. Jhe, Phys. Rev. A 44, 5932 (1991).

[21] E.A. Hinds, Adv. At. Mol. Opt. Phys. 28, 237 (1991).

[22] E.A. Hinds, Adv. At. Mol. Opt. Phys. Suppl. 2, 1 (1994).

[23] S. A. Ellingsen, S.Y. Buhmann, and S. Scheel, Phys. Rev. A 80, 022901 (2009).

[24] S. Å. Ellingsen, Y. Sherkunov, S.Y. Buhmann, and S. Scheel, in Proceedings of Ninth Conference on Quantum Field Theory Under the Influence of External Conditions (QFEXT09) edited by M. Bordag and K. A.
Milton (World Scientific, 2010), p. 168, Preprint: quant-ph/0910.5608.

[25] J. A. Crosse, S. Å. Ellingsen, K. Clements, S. Y. Buhmann, and S. Scheel, accepted for publication in Phys. Rev. A (2010); Preprint: quant-ph/1005.2485.

[26] S. Scheel and S. Y. Buhmann, Phys. Rev. A 80, 042902 (2009).

[27] M. Levin, A.P. McCauley, A.W. Rodriguez, M.T.H. Reid, S.G. Johnson, Preprint: cond-mat/1003.3487.

[28] S.Å. Ellingsen, S. Y. Buhmann, and S. Scheel, Phys. Rev. Lett. 104, 223003 (2010).

[29] D. Kleppner, Phys. Rev. Lett. 47, 233 (1981).

[30] P. Goy, J. M. Raimond, M. Gross, and S. Haroche, Phys. Rev. Lett. 50, 1903 (1983).

[31] M. S. Ünlü and S. Strite, J. Appl. Phys. 78, 607 (1995).

[32] H. Walther, B. T. H. Varcoe, B.-G. Englert, and T. Becker, Rep. Prog. Phys. 69, 1325 (2006).

[33] L.-W. Li, M.-S. Leong, T.-S. Yeo, and P.-S. Kooi, J. Electromag. Waves Appl. 14, 961 (2000).

[34] C.-T. Tai, Dyadic Green's Functions in Electromagnetic Theory (Scranton: Intext Educational Publishers, 1971), Ch. 6.

[35] A. Lambrecht and S. Reynaud, Eur. Phys. J., D 8, 309 (2000).

[36] I. Brevik, S. A. Ellingsen, and K. A. Milton, New J. Phys. 8, 236 (2006).

[37] M. Bordag, G. L. Klimchitskaya, U. Mohideen, and V. M. Mostepanenko, Advances in the Casimir effect (Oxford University Press, Oxford, 2009).

[38] R. S. Decca, D. López, E. Fischbach, G. L. Klimchitskaya, D. E. Krause, and V. M. Mostepanenko, Ann. Phys. 318, 37 (2005).

[39] S. Y. Buhmann, S. Scheel and J. Babington, Phys. Rev. Lett. 104, 070404 (2010).

[40] M. Abramowitz and I. A. Stegun Handbook of Mathematical Functions (New York: Dover, 1964).

[41] S. Y. Buhmann, M. R. Tarbutt, S. Scheel, and E. A. Hinds, Phys. Rev. A, 78, 052901 (2008).

[42] One may note here that the original pole due to the zero of $J_{m}(x)$ in the prefactor (2.15) is cancelled by the presence of $\tilde{\jmath}^{2}$ in the denomitor whereas $\tilde{\jmath}$ only enters to linear order in the numerator. This is how the pole is moved to the value of $k R$ which solves Eq. (3.7). 